\title{
Periodic Solution for Neutral Type Neural Networks
}

\author{
Wenxiang Zhang, Yan Yan, Zhanji Gui, Kaihua Wang* \\ School of Mathematics and Statistics, Hainan Normal University, Haikou, Hainan \\ Email: *kaihuawang@qq.com
}

Received 2013

\begin{abstract}
The principle aim of this paper is to explore the existence of periodic solution of neural networks model with neutral delay. Sufficient and realistic conditions are obtained by means of an abstract continuous theorem of k-set contractive operator and some analysis technique.
\end{abstract}

Keywords: Neutral-type Neural Networks; k-Set Contractive Operator; Periodic Solution

\section{Introduction}

Man-made neural networks have been widely used in the fields of pattern recognition, image processing, association, optimal computation, and others. However, owing to the unavoidable finite switching speed of amplifiers, time delays in the electronic implementations of analog neural networks are inevitable, which may cause undesirable dynamic network behaviors such as oscillation and instability. Thus, it is very important to investigate the dynamics of delay neural networks.

The existence of periodic oscillatory solutions of neural networks model has been studied by many researchers [1-6]. Some authors [3-5] used the well-known Hopf bifurcation theory to discuss the bifurcating periodic solutions. However, the usual Hopf bifurcation theory cannot be applied to non-autonomous system. In [6], $\mathrm{Li}$ and $\mathrm{Lu}$ applied the theory of coincidence degree to non-autonomous neural networks system and obtained some new criteria for the existence of periodic solutions.

We consider the following model for neutral type neural networks with periodic coefficient:

$$
\dot{x}_{i}(t)=-a_{i}(t) x_{i}(t)+\sum_{j=1}^{n} b_{i j}(t) g_{j}\left(\dot{x}_{j}\left(t-\tau_{i j}(t)\right)\right)+I_{i}(t),
$$

where $n$ is the number of neurons in the network, $x_{i}(t)$ $(i=1,2, \ldots, n)$ corresponds to the state of the $i$ th unit at time $t, a_{i}(t)$ denotes the neuron firing rate, $b_{i j}(t)$ represents the neutral delayed connection weight.

$$
g(x)=\left(g_{1}\left(x_{1}\right), g_{2}\left(x_{2}\right), \ldots, g_{n}\left(x_{n}\right)\right)^{T}: R^{n} \rightarrow R^{n}
$$

is the activation function. $I(t)=\left(I_{1}(t), I_{2}(t), \ldots, I_{n}(t)\right)^{T}$ is the $\omega$-periodic external input to the $i$ th neuron.

Throughout this paper, we assume that:

${ }^{*}$ Corresponding author.
(H1) Functions $g_{j}(u)(j=1,2, \ldots, n)$ are globally Lipschitz continuous with the Lipschitz constant $L_{j}>0$, that is, $\quad\left|g_{j}\left(u_{1}\right)-g_{j}\left(u_{2}\right)\right| \leq L_{j}\left|u_{1}-u_{2}\right|$, for all $u_{1}, u_{2} \in R$.

(H2) Functions $\tau_{i j}(t) \quad(i, j=1,2, \ldots, n)$ are nonnegative, bounded and continuously differentiable defined on $R^{+}$and $\dot{\tau}_{i j}<1$, where $\dot{\tau}_{i j}(t)$ express the derivative of $\tau_{i j}(t)$.

(H3) There exist constant $\alpha_{j} \geq 0, \beta_{j}>0$, such that $\forall x \in R,\left|g_{j}(x)\right| \leq \alpha_{j}|x|+\beta_{j}, \quad j=1,2, \ldots, n$.

Remark 1.1 It is easy to verify that if condition (H1) is satisfied then condition (H3) holds.

The organization of this paper is as follows. Preliminaries will be given in the next section. In section 3, we will study the existence of periodic solutions of system (1.1) by the abstract continuous theorem of k-set contractive operator.

\section{Preliminaries}

In order to study Equation (1.1), we should make some preparations. Let $E$ be a Banach space. For a bounded subset $A \subset E$, let

$$
\begin{aligned}
\alpha_{E}(A)= & \inf \{\delta>0 \mid \text { there is a finite number of } \\
& \text { subsets } A_{i} \subset A, \text { such that } A=\cup_{i=1} A_{i}, \\
& \text { and } \left.\operatorname{diam}\left(A_{i}\right) \leq \delta\right\}
\end{aligned}
$$

denotes the (Kuratoskii) measure of noncompactness, where $\operatorname{diam}\left(A_{i}\right)$ denotes the diameter of set $A_{i}$. Let $X$, $Y$ be two Banach spaces and $\Omega$ be a bounded open subset of $X$. A continuous and bounded map $N: \bar{\Omega} \rightarrow Y$ is called k-set contractive if for any bounded set $A \subset \Omega$ we have $\alpha_{Y}(N(A)) \leq k \alpha_{X}(A)$, where $k$ is a constant. In addition, for a Fredholm operator $L: X \rightarrow Y$ with index zero, according to [7], we may define 


$$
\begin{gathered}
l(L)=\sup \left\{r \geq 0 \mid r \alpha_{X}(A) \leq \alpha_{Y}(L(A)),\right. \text { for all } \\
\text { bounded subset } A \subset X\} .
\end{gathered}
$$

Lemma 2.1 Let $L: X \rightarrow Y$ be a Fredholm operator with index zero, and $a \in Y$ be a fixed point. Suppose that $N: \Omega \rightarrow Y$ is a k-set contractive with $k<l(L)$, where $\Omega \subset X$ is bounded, open, and symmetric about $0 \in \Omega$. Furthermore, we also assume that

1) $L x \neq \lambda N x+\lambda r$, for $x \in \partial \Omega, \quad \lambda \in(0,1)$;

2) $[Q N(x)+Q r, x] \cdot[Q N(-x)+Q r, x]<0$, for $x \in \operatorname{ker} L \cap \partial \Omega$, where $[\cdot, \cdot]$ is a bilinear form on $Y \times X$ and $Q$ is the project of $Y$ onto $\operatorname{Coker}(L)$.

Then there is a $x \in \bar{\Omega}$, such that $L x-N x=r$.

In order to use Lemma (2.1) for studying Equation (1.1), we set

$$
\begin{aligned}
Y=C_{\omega}=\left\{x(t)=\left(x_{1}(t), \ldots, x_{n}(t)\right) \in C\left(R, R^{n}\right):\right. \\
\\
\left.x_{i}(t+\omega) \equiv x_{i}(t), i=1, \ldots, n\right\},
\end{aligned}
$$

with the norm defined by $|x|_{0}=\sum_{i=1}^{n} \max _{t \in[0, \omega]}\left|x_{i}(t)\right|$, and

$$
\begin{aligned}
X=C_{\omega}^{1}=\left\{x(t)=\left(x_{1}(t), \ldots, x_{n}(t)\right) \in C^{1}\left(R, R^{n}\right):\right. & \left.x_{i}(t+\omega) \equiv x_{i}(t), i=1, \ldots, n\right\},
\end{aligned}
$$

with the norm $\|x\|=\max \left\{|x|_{0},|\dot{x}|_{0}\right\}$. Then $C_{\omega}, C_{\omega}^{1}$ are all Banach spaces. Let $L: C_{\omega}^{1} \rightarrow C_{\omega}$ defined by

$$
\begin{gathered}
L x=\frac{d x}{d t}=\left(\dot{x}_{1}, \ldots, \dot{x}_{n}\right)^{T} ; N: C_{\omega}^{1} \rightarrow C_{\omega}, \\
N\left[\begin{array}{c}
x_{1} \\
\vdots \\
x_{n}
\end{array}\right]=\left[\begin{array}{c}
-a_{1}(t) x_{1}(t)+\sum_{j=1}^{n} b_{1 j}(t) g_{j}\left(\dot{x}_{j}\left(t-\tau_{1 j}(t)\right)\right) \\
\vdots \\
-a_{n}(t) x_{n}(t)+\sum_{j=1}^{n} b_{n j}(t) g_{j}\left(\dot{x}_{j}\left(t-\tau_{n j}(t)\right)\right)
\end{array}\right]
\end{gathered}
$$

It is easy to see from [8] that $L$ is a Fredholm operator with index zero. Clearly, Equation (1.1) has a $\omega$-periodic solution if and only if $L x=N x+r$ for some $x \in C_{\omega}^{1}$, where $r:=I(t)=\left(I_{1}(t), \ldots, I_{n}(t)\right)^{T}$.

Lemma 2.2 [9] The differential operator $L$ is a Fredholm operator with index zero, and satisfies $l(L) \geq 1$.

Lemma 2.3 If $k=\max \left\{\sum_{j=1}^{n} b_{i j}^{+} L_{j}, i=1, \ldots, n\right\}<1$, here $b_{i j}^{+}=\max _{t \in[0, \omega]}\left|b_{i j}(t)\right|, \quad N: \Omega \rightarrow C_{\omega}$ is a k-contractive map.

Proof. Let $A \subset \bar{\Omega}$ be a bounded subset and let $\eta=$ $\alpha_{C_{\omega}^{1}}(A)$. Then, for any $\varepsilon>0$, there is a finite family of subsets $A_{i}$ satisfying $A=\cup_{i=1} A_{i}$ with $\operatorname{diam}\left(A_{i}\right) \leq \eta+\varepsilon$. Now let

$$
V_{i}\left(t, x_{i}, y_{1}, \ldots, y_{n}\right)=a_{i}(t) x_{i}-\sum_{j=1}^{n} g_{j}\left(y_{j}\right) .
$$

Since $V_{i}\left(t, x_{i}, y_{1}, \ldots, y_{n}\right)$ are uniformly continuous on any compact subset of $R \times R^{n+1}, A$ and $A_{i}$ are precompact in $C_{\omega}^{0}$, it follows that there is a finite family of sub- sets $A_{i j}$ of $A_{i}$ such that $A_{i}=\cup_{j=1} A_{i j}$ with

$$
\begin{gathered}
\mid V_{i}\left(t, x_{i}(t), \dot{u}_{1}\left(t-\tau_{i 1}(t)\right), \ldots, \dot{u}_{n}\left(t-\tau_{\text {in }}(t)\right)\right) \\
-V_{i}\left(t, u_{i}(t), \dot{u}_{1}\left(t-\tau_{i 1}(t)\right), \ldots, \dot{u}_{n}\left(t-\tau_{i n}(t)\right)\right) \mid \leq \varepsilon,
\end{gathered}
$$

for any $x, u \in A_{i j}$. Therefore we have

$$
\begin{aligned}
& |N x-N u|_{0} \\
= & \sup _{t \in[0, \omega]} \mid V_{i}\left(t, x_{i}(t), \dot{x}_{1}\left(t-\tau_{i 1}(t)\right), \ldots, \dot{x}_{n}\left(t-\tau_{i n}(t)\right)\right) \\
& -V_{i}\left(t, u_{i}(t), \dot{u}_{1}\left(t-\tau_{i 1}(t)\right), \ldots, \dot{u}_{n}\left(t-\tau_{i n}(t)\right)\right) \mid \\
\leq & \sup _{t \in[0, \omega]} \mid V_{i}\left(t, x_{i}(t), \dot{x}_{1}\left(t-\tau_{i 1}(t)\right), \ldots, \dot{x}_{n}\left(t-\tau_{i n}(t)\right)\right) \\
& -V_{i}\left(t, x_{i}(t), \dot{u}_{1}\left(t-\tau_{i 1}(t)\right), \ldots, \dot{u}_{n}\left(t-\tau_{i n}(t)\right)\right) \mid \\
& +\sup _{t \in[0, \omega]} \mid V_{i}\left(t, x_{i}(t), \dot{u}_{1}\left(t-\tau_{i 1}(t)\right), \ldots, \dot{u}_{n}\left(t-\tau_{i n}(t)\right)\right) \\
& -V_{i}\left(t, u_{i}(t), \dot{u}_{1}\left(t-\tau_{i 1}(t)\right), \ldots, \dot{u}_{n}\left(t-\tau_{i n}(t)\right)\right) \mid \\
\leq & \sum_{j=1}^{n} b_{i j}^{+}\left|g_{j}\left(\dot{x}_{j}\left(t-\tau_{i j}(t)\right)\right)-g_{j}\left(\dot{u}_{j}\left(t-\tau_{i j}(t)\right)\right)\right|+\varepsilon \\
\leq & \sum_{j=1}^{n} b_{i j}^{+} L_{j}\left|\dot{x}_{j}\left(t-\tau_{i j}(t)\right)-\dot{u}_{j}\left(t-\tau_{i j}(t)\right)\right|+\varepsilon \\
\leq & k \eta+(k+1) \varepsilon .
\end{aligned}
$$

As $\varepsilon$ is arbitrary small, it is easy to see that $\alpha_{C_{o}^{0}}(N(A)) \leq k \alpha_{C_{o}^{1}}(A)$.

Lemma 2.4 Let $\Phi \in C_{\omega}^{0}, \tau \in C_{\omega}^{1}$, and $\dot{\tau}(t)<1$, then $\Phi(v(t)) \in C_{\omega}^{0}$, where $v(t)$ is the inverse function of $t-\tau(t)$.

Throughout this paper, we assume that $\tau_{i j} \in C_{\omega}^{1}$, $\dot{\tau}_{i j}(t)<1 \quad(i, j=1, \ldots, n)$. So that $\tau_{i j}(t)$ has a unique inverse, and we set $v_{i j}(t)$ to represent the inverse of function $t-\tau_{i j}(t)$. . Meanwhile, we denote

$$
\bar{h}:=(1 / \omega) \int_{0}^{\omega} h(s) d s \text { and }\|h\|_{2}:=\left(\int_{0}^{\omega}|h(s)| d s\right)^{1 / 2},
$$

\section{Main Results}

Set

$$
\begin{aligned}
& p_{i j}^{2}=\max \left\{\frac{1}{1-\dot{\tau}_{i j}\left(v_{i j}(t)\right)}: t \in R\right\}, \\
& A=\operatorname{diag}\left(a_{1}^{l}, a_{2}^{l}, \ldots, a_{n}^{l}\right), B=\left(\tilde{b}_{i j}\right)_{n \times n}, \tilde{b}_{i j}=b_{i j}^{m} \alpha_{j} p_{i j}, \\
& C=\operatorname{diag}\left(a_{1}^{m}, a_{2}^{m}, \ldots, a_{n}^{m}\right), H=\left(\tilde{h}_{i j}\right)_{n \times n}, \\
& \tilde{h}_{i}=\sum_{j=1}^{n} b_{i j}^{m} \beta_{j} \sqrt{\omega}+\left\|I_{i}\right\|_{2} .
\end{aligned}
$$

Theorem 3.1 Assume that (H1), (H2) hold, furthermore, assume that (H4) $A-B C$ is non-singular M-matrix, then Equation (1.1) has at least one positive $\omega$ periodic solution.

Proof. We consider the operator Equation

$$
L x=\lambda N x+\lambda r, \lambda \in(0,1)
$$


Corresponding to Equation (3.1), we have

$\dot{x}_{i}(t)=\lambda\left[-a_{i}(t) x_{i}(t)+\sum_{j=1}^{n} b_{i j}(t) g_{j}\left(\dot{x}_{j}\left(t-\tau_{i j}(t)\right)\right)+I_{i}(t)\right]$

Suppose that $\left(x_{1}(t), \ldots, x_{n}(t)\right)^{T}$ is a solution of system (3.2) for a parameter $\lambda \in(0,1)$. Multiplying the $i$ th Equation of system (3.2) by $x_{i}$ and integrating over $[0, \omega]$ gives

$$
\begin{aligned}
a_{i}^{l}\left\|x_{i}\right\|_{2}^{2} \leq & \int_{0}^{\omega} x_{i}(t)\left[\sum_{j=1}^{n} b_{i j}(t) g_{j}\left(\dot{x}_{j}\left(t-\tau_{i j}(t)\right)\right)+I_{i}(t)\right] d t \\
\leq & \int_{0}^{\omega} x_{i}(t)\left[\sum_{j=1}^{n} b_{i j}^{m} \alpha_{j}\left|\dot{x}_{j}\left(t-\tau_{i j}(t)\right)\right|+\sum_{j=1}^{n} b_{i j}^{m} \beta_{j}+I_{i}(t)\right] d t \\
\leq & \sum_{j=1}^{n} b_{i j}^{m} \alpha_{j}\left(\int_{0}^{\omega}\left|x_{i}(t)\right|^{2} d t\right)^{1 / 2}\left(\int_{0}^{\omega}\left|\dot{x}_{j}\left(t-\tau_{i j}(t)\right)\right|^{2} d t\right)^{1 / 2} \\
& +\sum_{j=1}^{n} b_{i j}^{m} \beta_{j} \sqrt{\omega}\left(\int_{0}^{\omega}\left|x_{i}(t)\right|^{2} d t\right)^{1 / 2} \\
& +\left(\int_{0}^{\omega}\left|x_{i}(t)\right|^{2} d t\right)^{1 / 2}\left(\int_{0}^{\omega}\left|I_{i}(t)\right|^{2} d t\right)^{1 / 2} .
\end{aligned}
$$

And according to Lemma 2.4, we have

$$
\begin{aligned}
& \sqrt{\int_{0}^{\omega}\left|\dot{x}_{j}\left(t-\tau_{i j}(t)\right)\right|^{2} d t} \\
= & \sqrt{\int_{0}^{\omega} \frac{\left|\dot{x}_{j}(s)\right|^{2}}{1-\dot{\tau}_{i j}\left(v_{i j}(s)\right)} d s} \\
\leq & p_{i j}\left\|\dot{x}_{j}\right\|_{2} .
\end{aligned}
$$

Thus

$$
a_{i}^{l}\left\|x_{i}\right\|_{2} \leq \sum_{j=1}^{n} b_{i j}^{m} \alpha_{j} p_{i j}\left\|\dot{x}_{j}\right\|_{2}+\sum_{j=1}^{n} b_{i j}^{m} \beta_{j} \sqrt{\omega}+\left\|I_{i}\right\|_{2}
$$

Then we may rewrite Equation (3.3) as

$$
A X \leq B Y+H
$$

where $X=\left(\left\|x_{1}\right\|_{2}, \ldots,\left\|x_{n}\right\|_{2}\right)^{T}, \quad Y=\left(\left\|\dot{x}_{1}\right\|_{2}, \ldots,\left\|\dot{x}_{n}\right\|_{2}\right)^{T}$.

Multiplying the $i$ th Equation of system (3.2) by $\dot{x}_{i}$ and integrating over $[0, \omega]$ gives

$$
\begin{aligned}
\left\|\dot{x}_{i}\right\|_{2}^{2} & \leq \int_{0}^{\omega} \dot{x}_{i}(t)\left[\begin{array}{l}
-a_{i}(t) x_{i}(t) \\
\left.+\sum_{j=1}^{n} b_{i j}(t) g_{j}\left(\dot{x}_{j}\left(t-\tau_{i j}(t)\right)\right)+I_{i}(t)\right] d t \partial,
\end{array}\right] d t, \\
& \leq \int_{0}^{\omega} \dot{x}_{i}(t)\left[\begin{array}{l}
a_{i}^{m}\left|x_{i}(t)\right|+\sum_{j=1}^{n} b_{i j}^{m} \alpha_{j}\left|\dot{x}_{j}\left(t-\tau_{i j}(t)\right)\right| \\
+\sum_{j=1}^{n} b_{i j}^{m} \beta_{j}+I_{i}(t)
\end{array}\right] \\
& \leq a_{i}^{m}\left(\int_{0}^{\omega}\left|\dot{x}_{i}(t)\right|^{2} d t\right)^{1 / 2}\left(\int_{0}^{\omega}\left|x_{i}(t)\right|^{2} d t\right)^{1 / 2} \\
& +\sum_{j=1}^{n} b_{i j}^{m} \alpha{ }_{j}\left(\int_{0}^{\omega}\left|\dot{x}_{i}(t)\right|^{2} d t\right)^{1 / 2}\left(\int_{0}^{\omega}\left|\dot{x}_{j}\left(t-\tau_{i j}(t)\right)\right|^{2} d t\right)^{1 / 2} \\
& +\sum_{j=1}^{n} b_{i j}^{m} \beta_{j} \sqrt{\omega}\left(\int_{0}^{\omega}\left|\dot{x}_{i}(t)\right|^{2} d t\right)^{1 / 2} \\
& +\left(\int_{0}^{\omega}\left|\dot{x}_{i}(t)\right|^{2} d t\right)^{1 / 2}\left(\int_{0}^{\omega}\left|I_{i}(t)\right|^{2} d t\right)^{1 / 2} .
\end{aligned}
$$

That is,

$$
\begin{aligned}
\left\|\dot{x}_{i}\right\|_{2} \leq & a_{i}^{m}\left\|x_{i}\right\|_{2}+\sum_{j=1}^{n} b_{i j}^{m} \alpha_{j} p_{i j}\left\|\dot{x}_{j}\right\|_{2} \\
& +\sum_{j=1}^{n} b_{i j}^{m} \beta_{j} \sqrt{\omega}+\left\|I_{i}\right\|_{2}
\end{aligned}
$$

Then we may rewrite Equation (3.5) as

$$
Y \leq C X+H \text {. }
$$

Substituting (3.6) into (3.4), we obtain

$$
(A-B C) X \leq B H+H \text {. }
$$

It follows from Lemma 2.2 of paper [10] that $X \leq H^{+} \triangleq\left(h_{1}^{+}, \ldots, h_{n}^{+}\right)^{T}$. That is

$$
\left\|x_{i}\right\|_{2} \leq h_{i}^{+}, i=1, \ldots, n,
$$

where $H^{+} \triangleq\left(h_{1}^{+}, \ldots, h_{n}^{+}\right)^{T}=(A-B C)^{-1}(B H+H)$

Substituting (3.8) into (3.6), we obtain

$$
\left\|x_{i}\right\|_{2} \leq h_{i}^{+}, i=1, \ldots, n \text {. }
$$

It is easy to see that there exist two positive constants $N_{i}(i=1,2)$ such that

$$
|x|_{0}<N_{1},|\dot{x}|_{0}<N_{2} .
$$

Let $\Omega=\left\{x \in C_{\omega}^{1}:|x|_{0}<N_{1},|\dot{x}|_{0}<N_{2}\right\}$, and define a bounded bilinear form $[\cdot, \cdot]$ on $C_{\omega} \times C_{\omega}^{1}$ by

$$
[y, x]=\int_{0}^{\omega} y(t) x(t) d t .
$$

Also we define $Q: y \rightarrow \operatorname{Coker}(L)$ by $y \rightarrow \int_{0}^{\omega} y(t) d t$. Obviously,

$$
\{x \mid x \in \operatorname{ker} L \cap \partial \Omega\}=\left\{x \mid x \equiv N_{1}, \text { or } x \equiv-N_{1}\right\} .
$$

Without loss of generality, we may assume that $x \equiv N_{1}$. Thus

$$
\begin{aligned}
& {[Q N(x)+Q r, x] \cdot[Q N(-x)+Q r, x]=\omega^{2} \times N_{1}{ }^{2}} \\
& \times\left[\begin{array}{c}
{\left[-\bar{a}_{1} N_{1}+\sum_{j=1}^{n} \bar{b}_{1 j} g_{j}(0)+\bar{I}_{1}\right]\left[\bar{a}_{1} N_{1}+\sum_{j=1}^{n} \bar{b}_{1 j} g_{j}(0)+\bar{I}_{1}\right]} \\
\vdots \\
{\left[-\bar{a}_{n} N_{1}+\sum_{j=1}^{n} \bar{b}_{n j} g_{j}(0)+\bar{I}_{n}\right]\left[\bar{a}_{n} N_{1}+\sum_{j=1}^{n} \bar{b}_{n j} g_{j}(0)+\bar{I}_{n}\right]}
\end{array}\right] \\
& \text { If } N_{1}>\max _{1 \leq i \leq n}\left\{\frac{\sum_{j=1}^{n} \bar{b}_{i j} \beta_{j}+\bar{I}_{i}}{\bar{a}_{i}}\right\}>0 \text {, then } \\
& \bar{a}_{i} N_{1}>\sum_{j=1}^{n} \bar{b}_{i j} \beta_{j}+\bar{I}_{i}>\sum_{j=1}^{n} \bar{b}_{i j} g_{j}(0)+\bar{I}_{i}
\end{aligned}
$$

So from (3.12) we get

$$
\begin{gathered}
{\left[Q N\left(x_{i}\right)+Q\left(r_{i}\right), x_{i}\right] \cdot\left[Q N\left(-x_{i}\right)+Q\left(r_{i}\right), x_{i}\right]<0,} \\
i=1, \cdots, n
\end{gathered}
$$

Therefore, by using Lemma 2.1, we obtain that Equation (1.1) has at least one positive $\omega$-periodic solution. The proof is complete. 


\section{Acknowledgements}

This work is supported by the Natural Sciences Foundation of China under Grant No. 60963025.

\section{REFERENCES}

[1] X. Y. Liu and J. D. Cao, "On Periodic Solution of Neural Networks via Differential Inclusions," Neural Networks, Vol. 22, No. 4, 2009, pp. 329-334.

doi: 10.1016/j.neunet.2008.11.003

[2] J. Zhang and Z. J. Gui, "Periodic Solutions of Nonautonomous Cellular Neural Networks with Impulses and Delays," Nonlinear Analysis: Real World Applications, Vol. 10, No. 3, 2009, pp. 1891-1903.

doi: 10.1016/j.nonrwa.2008.02.029

[3] K. Gopalsamy and I. Leung, "Delay Induced Periodicity in a Neural Netlet of Excitation and Inhibition," Physica D: Nonlinear Phenomena, Vol. 89, No. 3-4, 1996, pp. 395-426. doi:10.1016/0167-2789(95)00203-0

[4] L. P. Shayer and S. A. Campbell, "Stability, Bifurcation and Multistability in a System of Two Coupled Neurons with Multiple Delays," SIAM Journal on Applied Mathematics, Vol. 61, No. 2, 2000, pp. 673-700. doi:0.1137/S0036139998344015

[5] J. Wu, T. Faria and Y. S. Huang, "Synchronization and Stable Phase-locking in a Network of Neurons with
Memory," Mathematical and Computer Modeling, Vol. 30, No. 1-2, 1999, pp. 117-138.

doi:0.1016/S0895-7177(99)00120-X

[6] Y. Li and L. Lu, "Global Exponential Stability and Existence of Periodic Solution of Hopfield- type Neural Networks with Impulses," Physics Letters A, Vol. 333, No. 1-2, 2004, pp. 62-71. doi:0.1016/j.physleta.2004.09.083

[7] Z. J. Gui, S. J. Lu and W. G. Ge, "Existence of Periodic Solution of Two Competition Dynamics System with Neutral Delay and Several Deviating Arguments," Chinese Journal of Engrg. Mathematics, Vol. 22, 2005, pp. 703-711.

[8] R. E. Gaines and J. L. Mawhin, Coincidence Degree and Nonlinear Differential Equations, Springer-Verlag, Berlin, 1977.

[9] Z. Liu and Y. Mao, "Existence Theorem for Periodic Solutions of Higher Order Nonlinear Differential Equations," Journal of Mathematical Analysis and Appications, Vol. 216, No. 2, 1997, pp. 481-490. doi:.1006/jmaa.1997.5669

[10] Z. Liu and L. Liao, "Existence and Global Exponential Stability of Periodic Solution of Cellular Neural Networks with Time-varying Delays," Journal of Mathematical Analysis and Applications, Vol. 290, No. 1, 2004, pp. 247-262. doi:10.1016/j.jmaa.2003.09.052 